\title{
Familial occurrence of cavernous angiomata of the brain
}

\author{
J. V. CLARK \\ From the Neuropathology Laboratory, Department of Pathology, University of Edinburgh
}

SUMMARY A 27 year old man had attacks of violence for three years before he died in 1945. Necropsy revealed multiple cavernous angiomata of the brain. This man's daughter died in 1969 at the age of 28 years after a year of intermittent headaches. Necropsy showed a large cavernous angioma in the right temporal lobe. Until now the literature has contained only scanty circumstantial evidence for a familial occurrence of cavernous angiomata of the brain.

Cavernous angiomata of the brain are not common and a familial occurrence appears exceptional. Indeed a review of the literature has revealed only two reports that hint at a familial incidence (Kufs, 1928; Michael and Levin, 1936). Similar neurological symptoms, and in one case the radiological demonstration of calcified nodules in the brain, suggested similar underlying lesions in two and five members of the respective families reported by these authors. However, in each of these families only one patient was examined pathologically.

This is the first report in which the occurrence of cavernous angiomata of the brain in two members of the same family has been confirmed pathologically.

\section{CASE REPORTS}

CASE 1 (Neuropathology no. 2910). The patient, a chef, was first seen at the Royal Infirmary, Edinburgh, on 2 January 1945 when he was aged 27 years. He was said to have had meningitis when he was 6 and had suffered from headaches since. In 1942 he was discharged from the army after a 10-week illness that consisted mainly of vomiting. During 1943 and 1944 he had several episodes of violence in which he was noisy and threw furniture about. He lost consciousness once. In June 1944 his wife separated from him and took their child, a daughter then aged 3 years, with her.

On examination the optic discs showed slight pallor but there were no visual field defects. The right pupil was larger than the left and the reactions to light and convergence were sluggish on both sides. There was slight nystagmus on looking to the left and the corneal reflexes were depressed. Lumbar puncture surprisingly showed an initial pressure of $220 \mathrm{~mm}$ cerebrospinal fluid but the fluid itself was otherwise normal. There were no other abnormal CNS signs. Advice that he should see anneurosurgeon was not accepted.
He was treated with phenobarbitone for his psychomotor attacks but in May 1945 he was readmitted to hospital because of impaired hearing. Examination showed severe bilateral ptosis, pin-point pupils, and severe limitation of eye movements. There was some degree of bilateral middle ear deafness but no gross facial weakness. Lumbar puncture revealed an initial pressure of $330 \mathrm{~mm}$ cerebrospinal fluid. Stereoradiograph views of the skull showed no abnormality. He now agreed to be seen by a neurosurgeon.

Operation was carried out on 23 May 1945 by $\mathrm{Mr}$. N. M. Dott. Ventriculography demonstrated a very extensive internal hydrocephalus with no filling of the aqueduct. Slight forwards displacement of the 3rd ventricle suggested a space-occupying lesion in the midbrain. A right parieto-occipital osteoplastic bone flap was raised and the approach was carried through the superior longitudinal fissure. An expanding lesion that could not be removed was present in the pineal neighbourhood and a Torkildsen tube was inserted to relieve the hydrocephalus.

Lumbar drainage was continued for some days and there was little if any improvement in his condition. He died suddenly on 4 June 1945 .

At necropsy the body was that of a well-developed, well-nourished male with a healed right occipital scalp incision. The bone flap was bulging and the skull showed well-marked erosion of the posterior clinoid processes. The dura, leptomeninges, and basal vessels appeared healthy. The cerebral hemispheres showed marked gyral flattening and the floor of the third ventricle was especially distended. Two areas of brownish discoloration each about $1.0 \mathrm{~cm}$ in diameter were present over the convolutions near the left temporal pole. No abnormalities were found in other systems other than purulent bronchiolitis. In particular there were no vascular lesions outside the brain.

Serial coronal slicing of the brain after fixation showed a $1.0 \mathrm{~cm}$ diameter angioma in the left inferior frontal 
gyrus (Fig. 1), a $1.5 \mathrm{~cm}$ diameter angioma with recent haemorrhage in the left temporal pole (Fig. 2), a $1.0 \mathrm{~cm}$ diameter angioma in the roof of the vestibule of the right lateral ventricle (Fig. 3), and a $2.5 \mathrm{~cm}$ diameter angioma replacing the posterior half of the midbrain (Fig. 3). This midbrain angioma lay chiefly behind and above the aqueduct, but also encircled it and there was a marked degree of hydrocephalus of the lateral and third ventricles.

Haematoxylin and eosin stained sections from the midbrain, frontal and ventricular angiomata showed numerous intercommunicating blood-filled cavernous spaces some of which were thrombosed (Fig. 4). Sections stained with Masson's trichrome technique superimposed on Hart's modification of Weigert's elastic stain showed

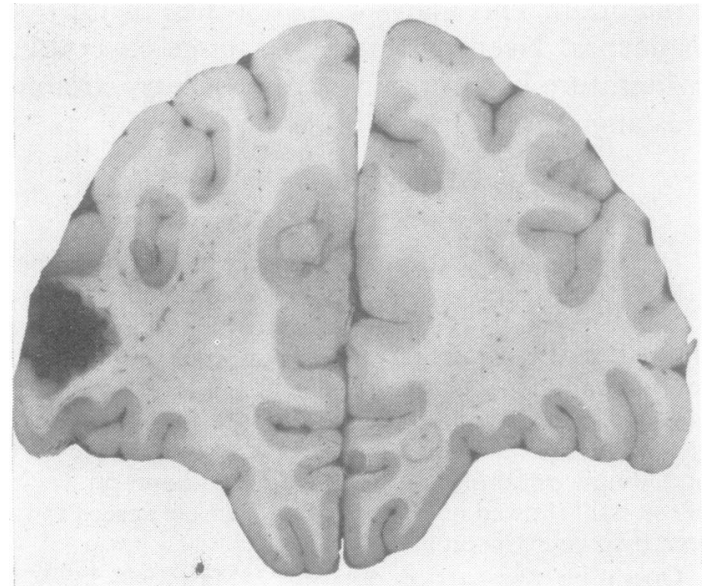

FIG. 1. Case 1. Coronal brain slice immediately anterior to genu showing angioma in left inferior frontal gyrus.

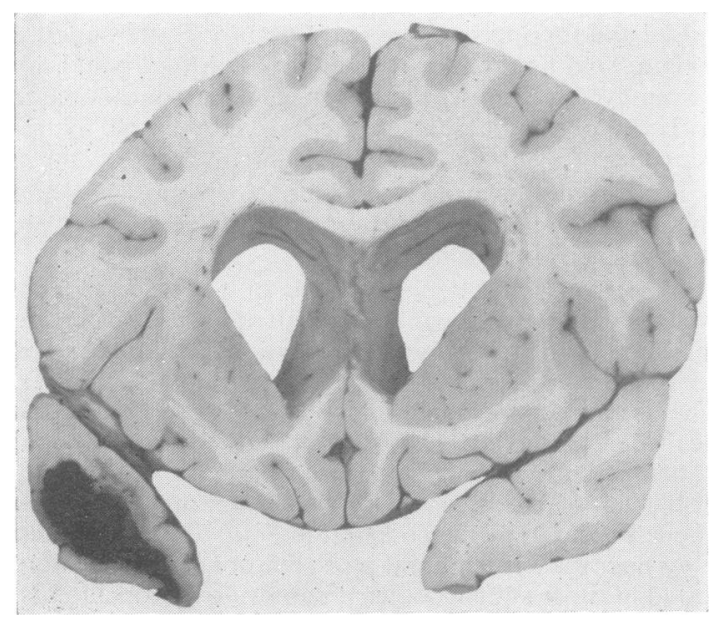

FIG. 2. Case 1. Angioma with haemorrhage in left temporal pole.

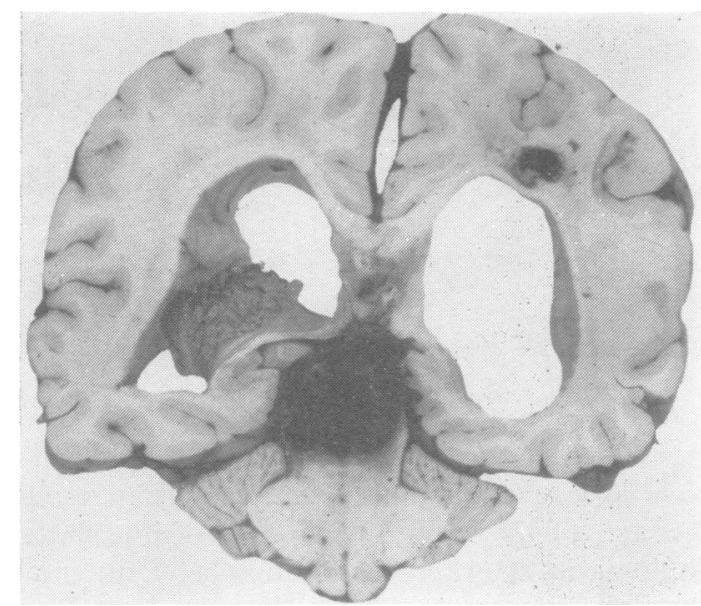

FIG. 3. Case 1. Angioma in roof of right lateral ventricle and angioma replacing posterior half of midbrain with production of hydrocephalus.

the walls of these vascular spaces to be composed entireए of fibrous tissue. Calcification was present in a few of the thicker walls and there was one area of ossification (Fig. 5). Around the lesions the cerebral tissue showed $\rightarrow$ gliosis and focal collections of haemosiderin containing

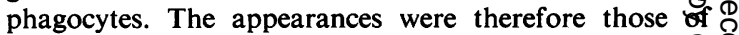
multiple cavernous angiomata with evidence of old ars recent haemorrhage.

CASE 2 (Neuropathology no. A 217/69). On 23 September 1969 the daughter of case 1, now a 28-year-old housewife, was admitted to hospital in a drowsy state. She was unable to give a history but it was ascertained that she had been having severe headaches since October 1968. These usually lasted about five days. No relief had been obtained from ergotamine or Anadin tablets. For five weeks before admission she suffered severe thumping headaches every morning and on two occasions these were preceded by a flashing zig-zag light in front of her left eye. For two or three days before admissio $n$ she had vomited on waking and on two occasions transient hypotension was recorded by her general practitioner.

On admission, examination showed photophobia but no neck stiffness and no papilloedema. She was given pethidine for pain. Fourteen hours after admission she had a cardiac arrest. The heart responded immediately to a thump on the chest but respiratory arrest persisted. She was placed on a respirator. Her pupils remained fixed and dilated and she died 18 hours after admission.

At necropsy performed 18 hours after death the body $ᄋ$ was that of a thin young woman of medium stature with no external abnormalities. The scalp, skull, and dural venous sinuses were normal but the dura was under tension. The leptomeninges were healthy but the cere- $N$ bral hemispheres were swollen, the gyri were flattened, and there was bulging of the floor of the third ventricle. 


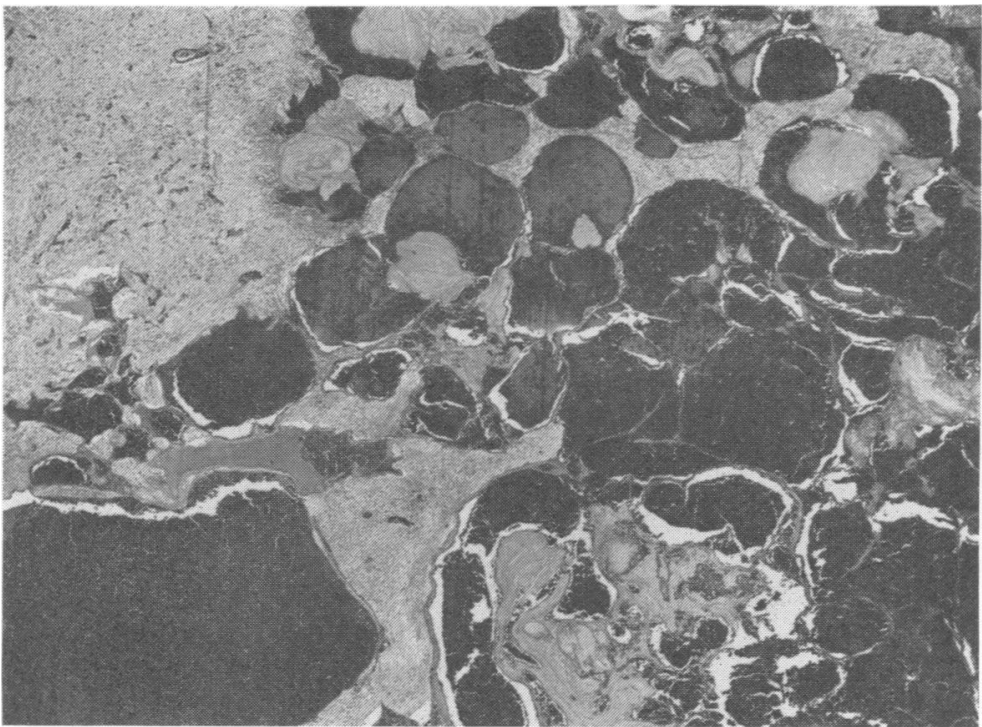

FIG. 4. Case 1. Numerous intercommunicating vascular spaces with thin walls composed entirely of fibrous tissue. Haematoxylin and eosin, $\times 12$.

All other systems were normal and in particular there were no vascular malformations outside the brain.

Examination of the brain after fixation showed, in addition to gyral flattening, bilateral nipping of the unci and marked cerebellar coning. The arteries at the base of the brain were healthy and the circle of Willis was normally constituted. Serial coronal slicing of the cerebrum revealed an angioma in the right temporal lobe which had recently bled (Fig. 6). Though closely related to the temporal horn, the angioma had not ruptured into the ventricular system. The angioma and haematoma together measured $5 \mathrm{~cm}$ in anteroposterior diameter by up to $4 \times 2.2 \mathrm{~cm}$ in maximal coronal dimensions at the level of the subthalamic nuclei (Fig. 7). Surrounding white matter showed yellowish brown discoloration, as did the ependymal lining of the compressed ventricles. There was shift of midline structures from right to left. No other angiomata were present in the cerebrum and slicing of the brain-stem and cerebellum showed no abnormalities apart from compression of the fourth ventricle.

Representative sections of the angioma were stained

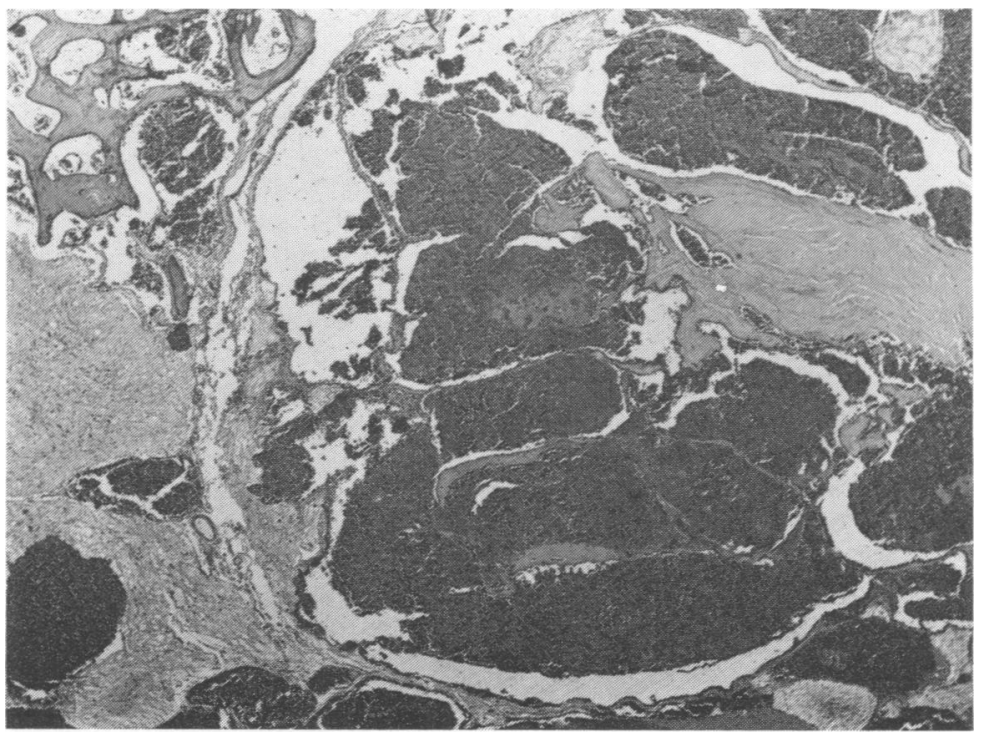

FIG. 5. Case 1. Osseous metaplasia (top left) in the cavernous angioma. Haematoxylin and eosin, $\times 25$. 


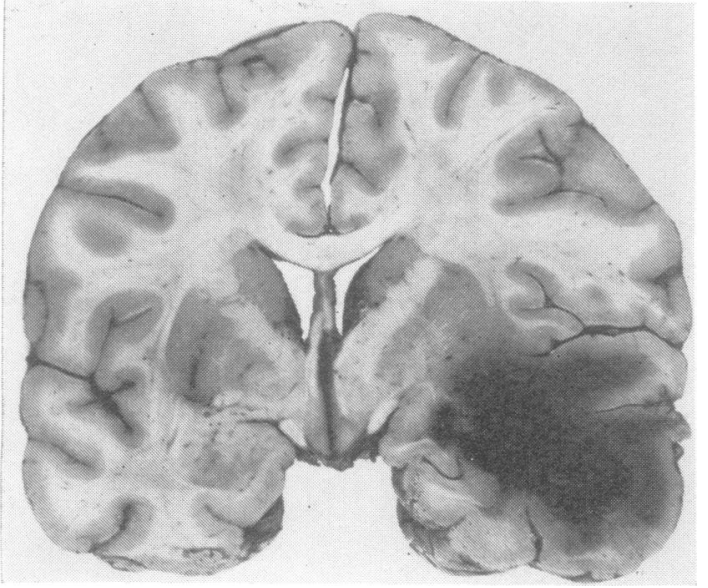

FIG. 6. Case 2. Coronal brain slice at level of mamillary bodies showing angioma in right temporal lobe. Surrounding white matter is stained by previous haemorrhage.

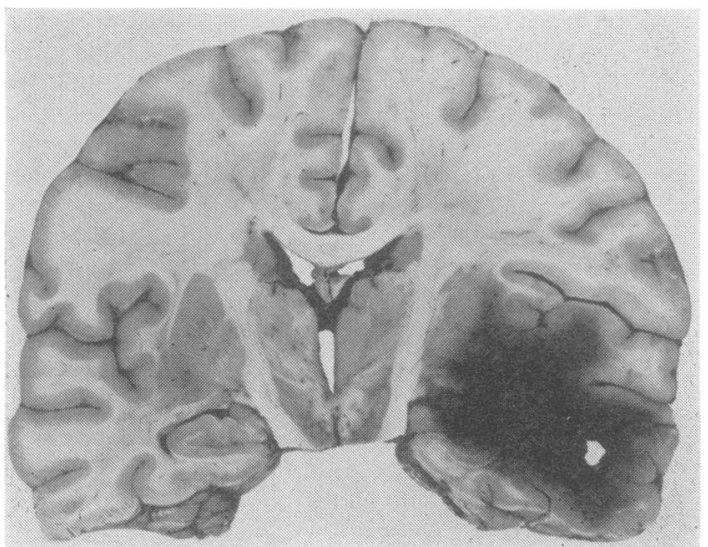

FIG. 7. Case 2. The angioma and haematoma at level of the subthalmic nuclei.

with haematoxylin and eosin, by the Hart-Masson technique, and by the Prussian blue reaction. Numerous contiguous small and large vascular spaces were observed (Fig. 8). The walls of these spaces showed considerable variation in thickness and were composed of collagen. This collagen was dense and sclerosed in places but there was no calcification or ossification. At the periphery of the lesion cavernous blood spaces were separated by gliotic tissue containing large amounts of haemosiderin and haematoidin pigment, mostly as granules in cerebral histiocytes. Fresh interstitial haemorrhages were also seen and some of the largest cavernous spaces were thrombosed. The lateral recess of the right temporal horn was compressed by the encircling angioma (Fig. 9) and the choroid plexus was adherent to the wall of the temporal horn in several places. The appearances suggested that the angioma might have acquired a secondary blood supply via the choroidal arteries.

The pathological diagnosis was therefore cavernous angioma of the right temporal lobe with old and recent haemorrhage.

FAMILY STUDY It is recorded that case 1 , when seen in 1945 , said there was no history of epilepsy in his family. A recent attempt to construct a family tree has been only partially successful but about 50 close relatives are in good health and there have been no other sudden deaths in the family as far as can be ascertained. Case 1 had four brothers who are all alive. Each has at least four children and many grand-children. Case 2 was the only child of case 1 . The two children of case 2 , now aged 8 and 6 years, are alive and well.

\section{DISCUSSION}

Noran (1945) reviewed 12 different classifications of intracranial vascular tumours and malformations, some of which included angioblastic meningioma, and he concluded by suggesting a classification of his $\dot{\sigma}$ own. Much of the confusion surrounding this subp $\infty$ ject in the earlier literature was due to the divere opinions as to which lesions were neoplasms an which malformations, and many reports gave descriptions and illustrations that failed to subp stantiate the diagnoses made by the authors.

Angioblastic meningioma and capillary haemo angioblastoma are now considered to be vascular $\stackrel{\Phi}{2}$ neoplasms. Vascular malformations of the centrat $\overrightarrow{0}$ nervous system are classified by Russell and Rubinstein (1963) thus:

Blood-vessel hamartomata

1. Capillary telangiectases

2. Cavernous angioma (cavernoma)

3. Venous and arteriovenous malformations

i. arteriovenous malformation

ii. venous malformation

iii. Sturge-Weber syndrome.

Most vascular malformations of the central nervous system may be placed into one of these distinct categories, though occasional examples show mixed features. Wyburn-Mason $(1943$, p. 49) presented evidence for grouping telangiectases and cavernous angiomata together. Certainly a distinction between the two may be difficult in a few instances (McCormick, 1966) but most cases can be separated into one or other type. Extracranial haemangiomata may occur in some patients with cavernous angiomata of the central nervous system (Wood, White, and Kernohan, 1957) but these cases are readily separable from the Rendu-Osler-Weber syndrome of hereditary telangiectasia. The evidence for hereditary factors in Sturge-Weber's disease is

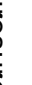

, \\ (n)}




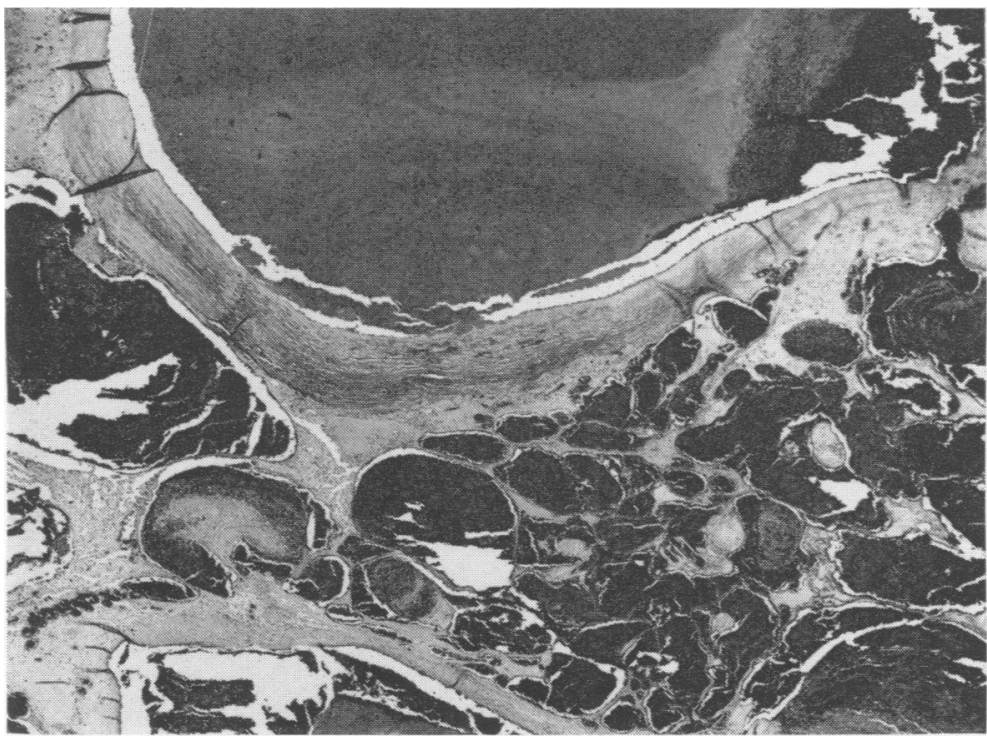

FIG. 8. Case 2. Cavernous angioma with features identical with those of case 1 (Fig. 4). Haematoxylin and eosin, $\times 12$.

inconclusive but the vascular lesion in this condition is a type of venous malformation that is quite different from a cavernous angioma. A familial incidence is established in von-Hippel-Lindau's disease (Nicol, 1957), but the capillary haemangioblastoma, a true neoplasm, is also quite distinct from the cavernous angioma.

Histological examination of the central nervous system vascular malformations from this man and his daughter showed the typical features of cavernous angiomata. Multiple cavernous angiomata were present in the father's brain but extracranial haemangiomata were not present in either patient. These two cases clearly indicate that cavernous angiomata, which are themselves uncommon, may rarely be familial. These lesions may be the cause of unheralded fatal haemorrhage as occurred in the two young people reported by Hawkins and Rewell

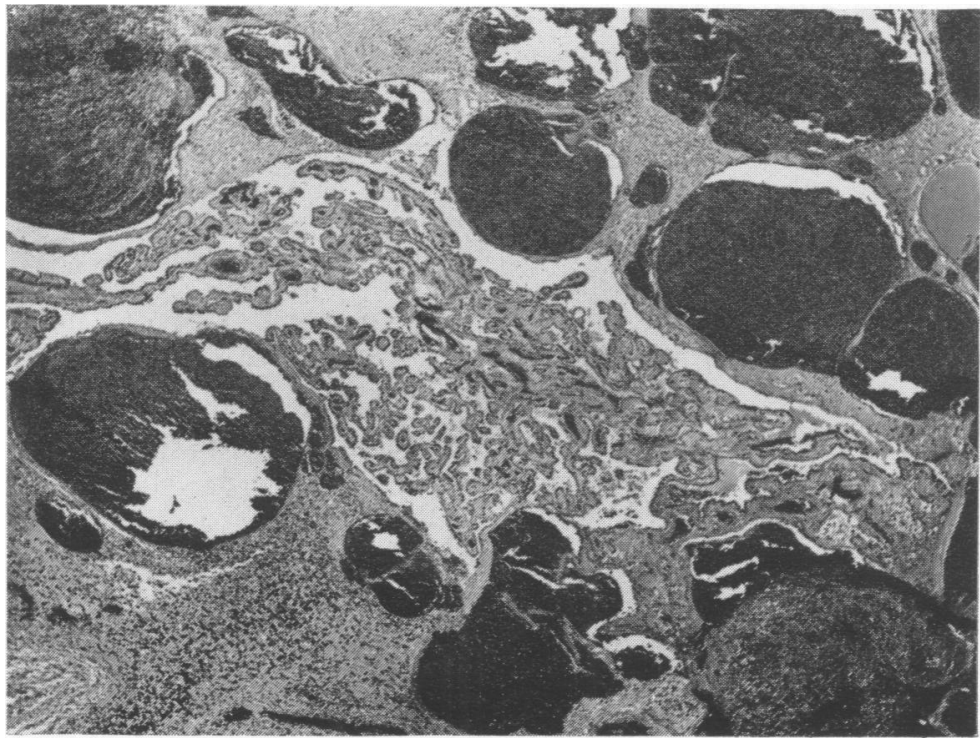

FIG. 9. Case 2. Right temporal horn with choroid plexus compressed by encircling cavernous angioma. Haematoxylin and eosin, $\times 25$. 
(1946), but if the diagnosis of cavernous angioma is made during life removal may be relatively easy (Schneider and Liss, 1958). Prodromal symptoms may be trivial as they were in the daughter (case 2) who complained only of headaches. Despite the rarity of a family history it may, when available, help to establish the diagnosis.

I wish to thank Professor N. M. Dott and Professor W. Blackwood for permission to publish the clinical and pathological details respectively of case 1 , and I wish to thank Dr. D. A. Seaton for permission to publish case 2. It is a particular pleasure to express my gratitude to Dr. A. F. J. Maloney for help and advice with the preparation of this paper.

\section{REFERENCES}

Hawkins, C. F., and Rewell, R. E. (1946). Unheralded, fatal haemorrhages in haemangiomata of the brain. Guy's Hosp. Rep., 95, 88-91.

Kufs, H. (1928). Uber heredofamiliäre Angiomatose des Gehirns und der Retina, ihre Beziehungen zueinander und zur Angiomatose der Haut. Zbl. ges. Neurol. Psychiat., 113, 651-686.

McCormick, W. F. (1966). The pathology of vascular ('arteriovenous') malformations. J. Neurosurg., 24, 807-816.

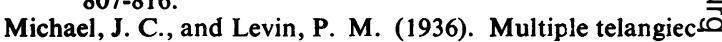
tases of the brain. A discussion of hereditary factors $T$ in their development. Arch. Neurol. Psychiat. (Chic.), 36, 514-529.

Nicol, A. A. McI. (1957). Lindau's disease in five generations. Ann. hum. Genet., 22, 7-15.

Noran, H. H. (1945). Intracranial vascular tumors and malformations. Arch. Path., 39, 393-416.

Russell, D. S., and Rubinstein, L. J. (1963). Pathology of Tumours of the Nervous System. 2nd edn., Edward Arnold: London.

Schneider, R. C., and Liss, L. (1958). Cavernous hem- $\overline{\bar{C}}$ angiomas of the cerebral hemispheres. J. Neurosurg., 15, 392-399.

Wood, M. W., White, R. J., and Kernohan, J. W. (1957). (ص) Cavernous hemangiomatosis involving the brain, spinal cord, heart, skin and kidney. Proc. Mayo Clin.,,$\vec{\circ}$ 32, 249-254.

Wyburn-Mason, R. (1943). The Vascular Abnormalities and $\vec{\omega}$ Tumours of the Spinal Cord and its Membranes. Henryo Kimpton: London. 